\title{
Comparison of Superconductors and Permanent Magnets for Small-scale Magnetic Resonance Imaging Devices
}

\author{
Insinga, A. R.; Christensen, J. J. L.; Abrahamsen, A. B.; Wulff, A. C.
}

Published in:

Applied Magnetic Resonance

Link to article, DOI:

10.1007/s00723-020-01202-4

Publication date:

2020

Document Version

Peer reviewed version

Link back to DTU Orbit

Citation (APA):

Insinga, A. R., Christensen, J. J. L., Abrahamsen, A. B., \& Wulff, A. C. (2020). Comparison of Superconductors and Permanent Magnets for Small-scale Magnetic Resonance Imaging Devices. Applied Magnetic Resonance, 51(6), 545-566. https://doi.org/10.1007/s00723-020-01202-4

\section{General rights}

Copyright and moral rights for the publications made accessible in the public portal are retained by the authors and/or other copyright owners and it is a condition of accessing publications that users recognise and abide by the legal requirements associated with these rights.

- Users may download and print one copy of any publication from the public portal for the purpose of private study or research.

- You may not further distribute the material or use it for any profit-making activity or commercial gain

- You may freely distribute the URL identifying the publication in the public portal 
See discussions, stats, and author profiles for this publication at: https://www.researchgate.net/publication/341357204

\section{Comparison of Superconductors and Permanent Magnets for Small-scale Magnetic Resonance Imaging Devices}

Article in Applied Magnetic Resonance · May 2020

DOI: $10.1007 / 500723-020-01202-4$

CITATIONS

READS

0

59

4 authors, including:

Andrea Roberto Insinga

Technical University of Denmark

17 PUBLICATIONS 128 CITATIONS

SEE PROFILE

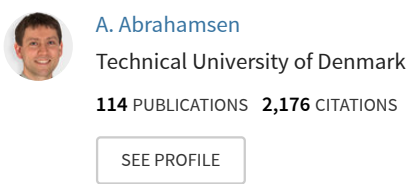

Anders Christian Wulff

Technical University of Denmark

25 PUBLICATIONS 140 CITATIONS

SEE PROFILE

Some of the authors of this publication are also working on these related projects:

INNWIND.EU View project

Substrates for coated conductors View project 


\title{
Comparison of superconductors and permanent magnets for small-scale magnetic resonance imaging devices
}

\author{
A. R. Insinga, J. J. L. Christensen, A. B. Abrahamsen, A. C. Wulff
}

March 30, 2020

\begin{abstract}
In this feasibility study we use finite element method simulations to compare superconductors (SC) coils and permanent magnets (PM) assemblies as main field source for a small-scale magnetic resonance imaging scanner. The motivation behind this investigation is that for certain tissues, e.g. knee ligament, diagnosis can be performed with equipment operating with relatively weak magnetic fields. It is then interesting to assess whether the relaxed field requirement enables alternative technologies, which present some advantages over the well-established helium-cooled low-temperature superconductors.

As prototypical magnetic systems we consider the Helmholtz coil and the Halbach cylinder for the SC and PM cases, respectively. Each possibility is simulated over a wide range of combinations of the fundamental design parameters. We estimate field strength, field homogeneity, and total cost over the service life of the device. From these figures of merit we evaluate the optimal combinations of design parameters. Finally we compare the SC and PM systems and we establish the range of field requirement values for which one alternative is more suitable than the other one.

The result of the present investigation provides the starting point for more detailed studies where more specific design considerations are taken into account.
\end{abstract}

\section{Introduction}

In this study we perform a cost comparison between superconductor (SC) coils and permanent magnet (PM) assembly field sources as main magnet of small-scale magnetic resonance imaging (MRI) apparatus.

The majority of commercial whole-body superconducting scanners typically work with field magnitudes of either 1.5 or $3 \mathrm{~T}$, and they use $\mathrm{NbTi}$ superconducting coils [13. Since NbTi is a low temperature superconductor and needs to be operated at temperatures typically around 2-4 K[19], liquid helium cooling is unavoidable. Seeing that helium prices have risen in recent years, and are expected to continue to rise due to an imbalance between demand and supply [16], alternative methods are being investigated. It should also be noted that the MRI system cost roughly scales as the volume of the warm bore [13], thus suggesting that a smaller system would be significantly more cost-effective for limb diagnosis than employing a full body scanner. Here we compare two viable alternatives: employing high-temperature superconductors (HTS) [17, 18] that have previously been successfully applied for making small MRI magnets, which do not require liquid helium to be operated, or permanent magnet (PM) assemblies, which do not require active cooling. 
Imaging quality in MRI depends primarily on field homogeneity and field strength. It is therefore critical that magnetic fields created are as homogeneous and powerful as possible. However, some diagnostic procedures may need a lower field strength and homogeneity than others. It is hence of interest to create a small MRI magnet for diagnosis of conditions affecting knee ligaments [15], since this application is expected to require lower precision than, e.g., diagnosing brains tumors. This would relax the requirement for high field magnets, since people who "only" need knee scans can be diagnosed with smaller and more cost-effective machines. Thus, in this work we will focus on small scale devices sized for the representative case of knee-ligament scanning.

A promising candidate from the HTS materials family for MRI systems are the Rare-Earth Barium-Copper-Oxide (REBCO) coated conductors, which have superior performance at high magnetic fields and temperatures [20]. They are however complex to fabricate, meaning they are relatively expensive, compared to the much cheaper NbTi. HTS tape is estimated to cost $22 \$ \mathrm{kA}^{-1} \mathrm{~m}^{-1}$ (at $30 \mathrm{~K}, 0.6 \mathrm{~T}$ ) [12, 9, while NbTi is estimated to cost $1.5 \$ \mathrm{kA}^{-1} \mathrm{~m}^{-1}$ (at $4.2 \mathrm{~K}$, $5 \mathrm{~T}$ ) 21. The cost difference makes it challenging for HTS to compete with the more mature technological field of NbTi-based MRI systems operating at low magnetic field intensities and elevated temperatures. The Helmholtz coil is the most basic and notable example of coil arrangement aimed at creating a homogeneous field distribution 14. Modern MRI devices are based on more advanced designs, typically constituted by more than six coils, that are optimized for the specific field and geometry constraints of each case [13.

Among modern permanent magnet materials, the most widely used are rare-earth based magnets, and particularly neodymium-iron-boron (NdFeB) magnets 26. The different commercially available grades reach values of remanent flux density up to $B_{r}=1.45 \mathrm{~T}$, and intrinsic coercivity up to $H_{c i}=2600 \mathrm{kA} / \mathrm{m}[29$. The Halbach cylinder is a well-known permanent magnet assembly which has the purpose of generating a homogeneous field [25]. It has been investigated for NMR [24, 23], and for MRI[10, but the larger volumes needed for MRI pose additional challenges.

The subject of the present work is thus an exploratory comparison of two alternatives as main magnetic field source for a small-scale MRI apparatus: a REBCO-based Helmholtz coil, or a NdFeB-based Halbach cylinder, as illustrated in Fig. 1. We compare field strength and field precision achievable with these two prototypical magnetic systems over a wide range of design parameters. We estimate the capital cost and operating cost of the two options and investigate the conditions where one of the two is more convenient than the other. The calculation of the magnetic field distribution corresponding to the different cases are performed by finite element methods analysis. As discussed in Sec. 2 our study is based on some simplifying approximations which are suitable for a preliminary investigation. In any case a detailed analysis of any real MRI machine should necessarily consider the specific design of the magnetic system optimized for the particular requirements under consideration.

\section{Methods}

The geometry of the magnetic systems is conveniently described by adopting a cylindrical coordinate system: we will denote the radial, angular and vertical coordinates by $r, \phi$, and $z$, respectively. The corresponding unit vectors will be denoted by $\hat{\boldsymbol{e}}_{r}, \hat{\boldsymbol{e}}_{\phi}$, and $\hat{\boldsymbol{e}}_{z}$. The vector pointing to a generic point of space will be denoted by $\boldsymbol{x}$.

The region of interest (ROI), is the region where the object to be scanned is placed. In this study the ROI is a cylinder with axis along the $z$ direction. Also reported in Tab. 1 the radius of the cylinder is $\tilde{R}=7.5 \mathrm{~cm}$ and the length is $\tilde{L}=15 \mathrm{~cm}$. These are realistic values for the case of an apparatus designed for scanning knee ligaments. 
It is worth mentioning that in many studies the region of interest is spherical instead of cylindrical. In this situation, it is conventional to express the size of the ROI by the diameter of spherical volume (DSV), which is the diameter of such sphere. The largest sphere that can be fitted in the cylindrical ROI considered in this paper has a DSV of $15 \mathrm{~cm}$. Since the cylindrical ROI encloses this sphere, the field-distortion is overestimated with respect to the value that would be obtained when considering the inscribed spherical region.

As mentioned in the introduction, MRI machines consist of complex magnetic systems composed of different parts. The main magnet has the purpose of generating an intense static field which must be as homogeneous as possible over the ROI. The gradient coils are operated during the scanning process by a time-varying current, and thus generating a time-varying field which superimposes with the static field generated by the the main coil. In this way the total field can be altered to provide spatial information. There are three sets of gradient coils, one for each orthogonal direction of a Cartesian system having one of the axis parallel to the field generated by the main magnet. Moreover, the magnetic system includes coils for generating and detecting the radio-frequency excitation signal, and passive and active shimming for small corrections to the magnetic field distribution aimed at increasing its homogeneity.

The direction of the main field for superconductors-based designs is along the longitudinal axis of the coil, here denoted by $z$. On the contrary, for designs based on the Halbach cylinder the main field is normal to the axis of the cylinder, similar to open MRI designs. This important difference naturally has implications on the design of the gradient coils and the other coils composing the magnetic system. However, a detailed analysis of such consequences goes beyond the scope of the present work. In this study we focus on the principal field requirements of the main magnet.

\subsection{Helmholtz Coil}

For the case of the superconducting flux source we consider the prototypical geometry known as Helmholtz coil. This arrangement consists of two identical coils separated by a distance that is equal to their radius, here denoted by $R$. In this study the common axis of the two coils is oriented along the $z$ direction, and the coils are assumed to be placed symmetrically with respect to the origin of the coordinate system. The origin is thus the point where the coils are intended to generate a uniform field, directed along the $z$ axis. In fact, the distance between the coil is selected so that the second derivative of the $z$ component of the field with respect to $z$ is cancelled. Since, because of symmetry, the first derivative is already null in the middle point, the second derivative is the first term in the Taylor expansion which contributes to the inhomogeneity of the field $[22$. This analytical calculation is performed for infinitely thin circular wires, for which the field distribution can be written in closed-form expression in terms of elliptic integrals.

In our study each coil is assumed to have a square cross section, with side length given by $W$. The geometry is shown in the cutaway illustration of Fig. 1a. The flux density distribution is shown in the half plane $x=0, y>0$. The field lines are plotted as thin black lines, and the intensity is indicated by the shade of grey: a darker shade indicates a stronger field. The region of interest is indicated by the central cylinder.

We simulated all the different combinations of radius $R$, coil width $W$, and operating temperature $T$, for a grid delimited by the ranges reported in Tab. 1. As we can see from the table, the smallest value of $R$ is $19 \mathrm{~cm}$; the space between $R$ and the external radius of the ROI, denoted by $\tilde{R}=7.5 \mathrm{~cm}$, is thus left free for gradient coils, radio-frequency coils and thermal insulation [21. The parameter grid includes data points corresponding to $N_{S C}=11466$ different simulations and the results are presented in Sec. 3 . 


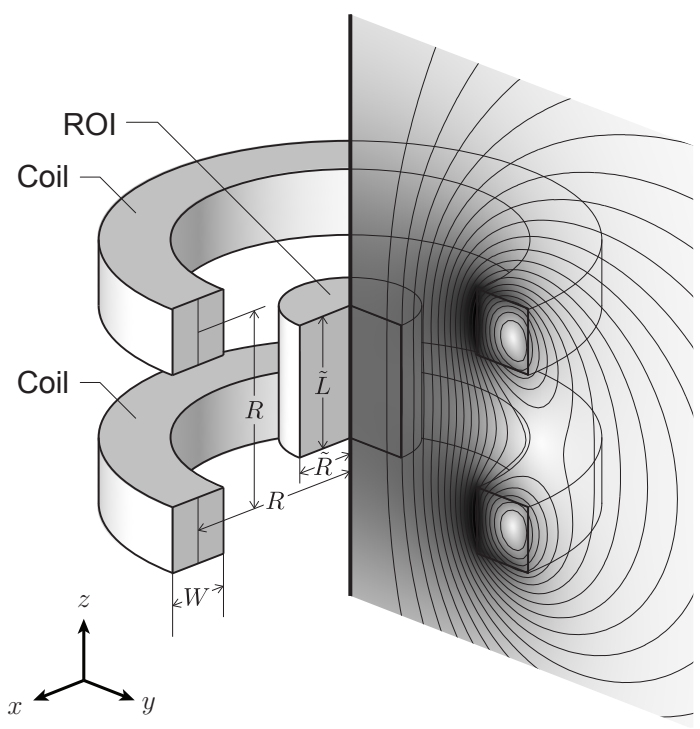

(a) Helmholtz Coil

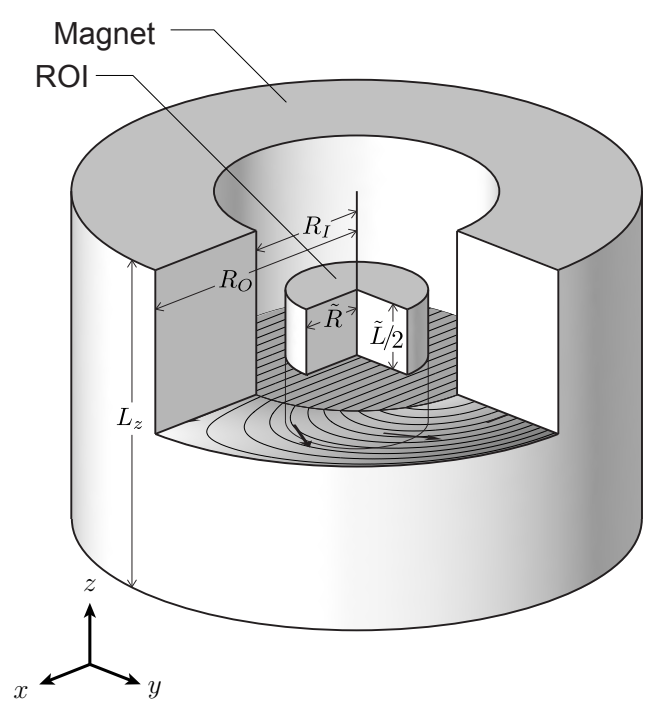

(b) Halbach Cylinder

Figure 1: Geometries of the two alternatives as main field-source for the MRI device. 1a Helmholtz coil cutaway illustration. The two coils, the region of interest (ROI) and the main design parameters are indicated. The field distribution is shown on an vertical plane: the field lines of $\boldsymbol{B}$ are shown and $\|\boldsymbol{B}\|$ is indicated by the brightness: a darker colour corresponds to a stronger field. $1 \mathrm{~b}$. Halbach cylinder cutaway illustration. For this case the field distribution is shown on a horizontal plane which bisects the ROI.

\subsection{Halbach Cylinder}

For the case of a permanent magnet flux source we consider the well known Halbach cylinder geometry [25]. This magnetic system consists of a hollow cylinder having internal radius $R_{I}$, external radius $R_{O}$, and axial length $L_{z}$. The direction of magnetization is given by

$$
\hat{\boldsymbol{e}}_{\|}=\cos (\phi) \hat{\boldsymbol{e}}_{r}+\sin (\phi) \hat{\boldsymbol{e}}_{\phi}
$$

This distribution has the purpose of generating a magnetic field oriented along the $x$ direction inside the bore of the cylinder. As can be seen in Fig. $1 \mathrm{~b}$, the $x$ direction is transverse to the axis of the cylinder. In the ideal case, corresponding to $L_{z} \rightarrow \infty$ and perfectly hard magnet, the field generated in the bore of the cylinder is perfectly uniform, the field outside the cylinder is exactly zero, and the magnitude of the flux density in the bore is given by [25]:

$$
B=B_{r} \log \left(\frac{R_{O}}{R_{I}}\right)
$$

where $B_{r}$ is the norm of the remanent flux density.

In practice the Halbach cylinder is realized by combining many blocks each characterized by uniform magnetization. The larger the number of blocks, the closer the magnetization distribution is to Eq. 1] A consequence of the segmentation is to reduce the average field magnitude in the bore, and also its homogeneity. However, in this study we do not include 


\begin{tabular}{|l|l|l|}
\hline Description & Symbol & Value / Range \\
\hline \hline Region of interest (ROI) radius & $\tilde{R}$ & $7.5 \mathrm{~cm}$ \\
\hline ROI axial length & $\tilde{L}$ & $15 \mathrm{~cm}$ \\
\hline \hline Coil Radius & $R$ & {$[19 \mathrm{~cm}, 60 \mathrm{~cm}]$} \\
\hline Coil side length & $W$ & {$[0.2 \mathrm{~cm}, 4 \mathrm{~cm}]$} \\
\hline Coil temperature & $T$ & {$[4.2 \mathrm{~K}, 77 \mathrm{~K}]$} \\
\hline Coil \# of simulations & $N_{S C}$ & 11466 \\
\hline \hline Halbach inner radius & $R_{I}$ & $\sigma \times 10 \mathrm{~cm}$ \\
\hline Halbach scale factor & $\sigma$ & {$[1,7.5]$} \\
\hline Halbach outer radius & $R_{O}$ & $R_{I} \times[1,15]$ \\
\hline Halbach axial length & $L_{z}$ & $R_{I} \times[2,10]$ \\
\hline Halbach \# of simulations & $N_{P M}$ & 6138 \\
\hline
\end{tabular}

Table 1: Values and ranges of the design parameters used for the simulations.

the effect of segmentation into blocks. Conversely, we do consider the effect of finite length in the axial direction and non-linear magnetic behaviour. As discussed in Ref. 11] the non-linear behaviour limits the maximum value of field attainable with this magnetic system.

The Halbach cylinder is shown in the cutaway illustration of Fig. $1 \mathrm{~b}$. The arrows represent the direction of magnetization given by Eq. 1. The flux density distribution is shown in the plane $z=0$ which bisects the ROI: the field lines are plotted as thin black lines and the magnitude of the flux density is indicated by the grey tone, a darker shade indicates a larger magnitude.

We simulated different combinations of $R_{I}, R_{O}$ and $L_{z}$, corresponding to a grid delimited by the ranges reported in Tab. 1 The parameter denoted by $\sigma$ is thus a geometrical scale factor multiplying the dimensions of the permanent magnet cylinder: $R_{I}, R_{O}$, and $L_{z}$, are all proportional to $\sigma$, while the dimensions of the ROI are constant, i.e. they do not depend on $\sigma$. As can be noticed from Tab. 1. when $\sigma=1$ the inner radius is given by $R_{I}=10 \mathrm{~cm}$ :

$$
\sigma=R_{I} /(10 \mathrm{~cm})
$$

The parameter grid includes data points corresponding to $N_{P M}=6138$ different simulations. The results are presented in Sec. 3

\subsection{Simulation}

In order to simulate the magnetic behaviour of the systems we employed finite element method analysis. The simulations have been performed using the magnetic fields interface of the commercial software Comsol multiphysics. Since we are interested in the magnetostatic analysis of the system, the governing equations are Gauss' law for magnetism

$$
\boldsymbol{\nabla} \cdot \boldsymbol{B}=0
$$

and Ampere's law

$$
\boldsymbol{\nabla} \times \boldsymbol{H}=\boldsymbol{J}
$$

Here $\boldsymbol{B}$ denotes the magnetic flux density expressed in teslas, $\boldsymbol{\nabla} \cdot \boldsymbol{B}$ denotes the divergence of $\boldsymbol{B}, \boldsymbol{H}$ denotes the magnetic field expressed in amperes per meter, $\boldsymbol{\nabla} \times \boldsymbol{H}$ denotes the curl of $\boldsymbol{H}$, and $\boldsymbol{J}$ denotes the electrical current density expressed in amperes per square meter. The 
problem is formulated in terms of the magnetic vector potential $\boldsymbol{A}$. The magnetic flux density defined from the vector potential automatically satisfies Gauss' law:

$$
\boldsymbol{B}=\boldsymbol{\nabla} \times \boldsymbol{A}
$$

The following equation relates $\boldsymbol{B}$ and $\boldsymbol{H}$ with the magnetization $\boldsymbol{M}$, which is also expressed in amperes per meter:

$$
\boldsymbol{B}=\mu_{0}(\boldsymbol{H}+\boldsymbol{M})
$$

where $\mu_{0}$ denotes the vacuum permeability. The general constitutive relation describing the magnetic behaviour of the materials can be expressed as an equation binding $\boldsymbol{M}$ to $\boldsymbol{B}$ :

$$
M=\boldsymbol{f}(\boldsymbol{B})
$$

In conclusion, the partial differential equation solved by the program is:

$$
\boldsymbol{\nabla} \times\left(\mu_{0}^{-1} \nabla \times \boldsymbol{A}-\boldsymbol{f}(\boldsymbol{\nabla} \times \boldsymbol{A})\right)=\boldsymbol{J}
$$

Superconductor - In principle, an exact description of the magnetic behaviour of the superconducting material would require a time-dependent simulation which couples the previous equations with Faraday's law of induction, $\boldsymbol{\nabla} \times \boldsymbol{E}=-\partial_{t} \boldsymbol{B}$, which governs the electrical field $\boldsymbol{E}$ arising in the superconducting material when it experiences a time-varying magnetic field. Such detailed approach also considers the non linear engineering $\boldsymbol{J}$ - $\boldsymbol{E}$ relation typical for a technical superconductor [8, 7]:

$$
\boldsymbol{J}=J_{e}\left(\frac{\|\boldsymbol{E}\|}{E_{c}}\right)^{1 / n} \frac{\boldsymbol{E}}{\|\boldsymbol{E}\|}, \quad \text { with } E_{c}=10^{-6} \mathrm{~V} / \mathrm{cm}
$$

The relation between $\boldsymbol{J}$ and $\boldsymbol{E}$ in a given point is also affected by the magnetic flux density $\boldsymbol{B}$ in that point since $J_{e}=J_{e}(\boldsymbol{B})$.

However, such a treatment is computationally intensive and goes beyond the scope of this work. Instead, we performed the load-line analysis employing a simplified approach: the current density is directly determined by the maximum value that is compatible with the norm of $\boldsymbol{B}$ inside the superconducting coil. The relation between electrical current and magnetic flux density is shown in Fig. 2a for different temperatures going from $T=4.2 \mathrm{~K}$ to $77 \mathrm{~K}$. These curves represent the engineering current density $J_{e}$, i.e. average current density of the whole wire, and have been obtained from Ref. [12 by assuming a wire-thickness of $80 \mu \mathrm{m}$. Since we assume that all the windings of each coil are connected in series, the value of current density selected for the simulations corresponds to the maximum value of $B$ experienced by the whole coil. Moreover, we applied an extra reduction factor of $\lambda=J / J_{e}=0.75$, meaning that the coil is operated well below the conditions for which the risk of quenching is significant. In conclusion, the current density inside the region $\mathcal{R}_{\text {Coil }}$ occupied by the coils, shown in Fig. $1 \mathrm{a}$ is given by

$$
\boldsymbol{J}=\lambda J_{e}\left(\max _{\mathcal{R}_{\text {Coil }}}(\|\boldsymbol{B}\|), T\right) \hat{\boldsymbol{e}}_{\phi}
$$

where the engineering current density $J_{e}(B, T)$ is the function of flux density and field represented by the curves shown in Fig. 2a. corresponding to a second generation HTS (2G HTS) advanced pinning (AP) wire developed at SuperPower Inc.. For practical reasons related to the implementation we fitted the data-points from Ref. [12] with a model function which has been used for the computations. More details are given in appendix A.1. For the Helmholtz coil we also assumed that the magnetization is zero everywhere.

Permanent magnet - The constitutive relation governing the behaviour of the permanent magnet along the easy axis of magnetization is shown in Fig. $2 \mathrm{~b}$ This curve has been extracted 
from the data reported in Ref. 29] for the grade N48 designated "Vacodym510", assuming that the operating temperature is $T=20^{\circ} \mathrm{C}$. As for the $J_{c}(B)$ relation, we also fitted the data from the reference with a model function, which is reported in appendix A.1. The magnetic behaviour along the hard axes is assumed to be the same as in vacuum: $B_{\perp}=\mu_{0} H_{\perp}$. Expressed as vector relation between $\boldsymbol{M}$ and $\boldsymbol{H}$, the constitutive relation is thus given by:

$$
\boldsymbol{M}=\hat{\boldsymbol{e}}_{\|} M_{\|}\left(\boldsymbol{H} \cdot \hat{\boldsymbol{e}}_{\|}\right)
$$

where $\hat{\boldsymbol{e}}_{\|}$is the direction defined in Eq. 1 and $M_{\|}$denotes the demagnetization branch of the $M-H$ hysteresis loop shown in Fig. 2b as a blue curve.

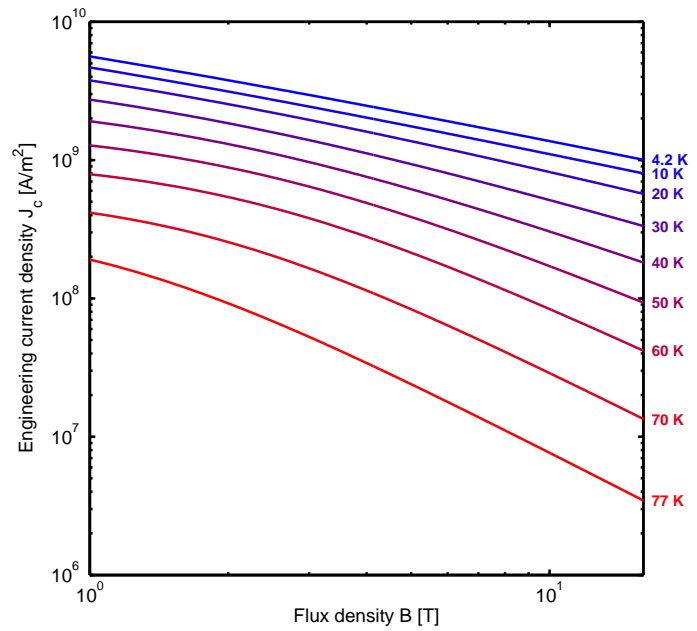

(a) Superconductor $J_{c}(B)$ relation

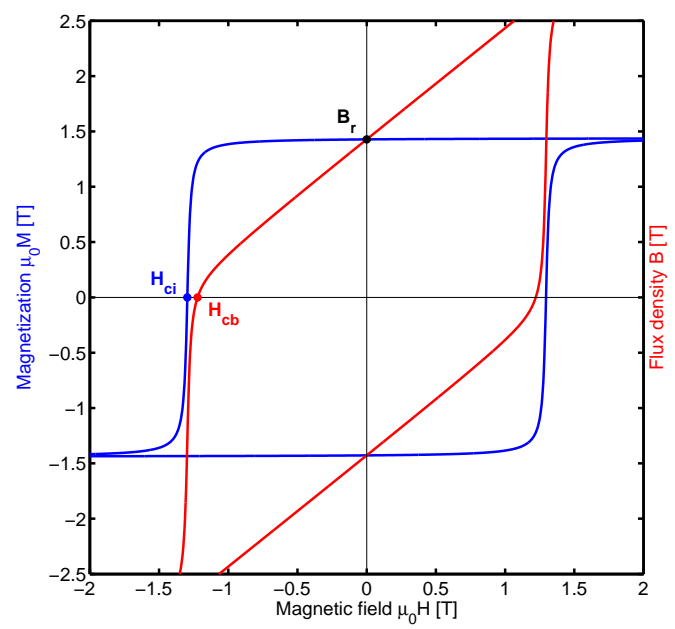

(b) Permanent magnet $M-H$ relation

Figure 2: Constitutive relations for the materials considered as magnetic field-source. 2a: REBCO superconductor engineering critical current density $J_{e}(B, T)$ relation for SuperPower Inc. 2G HTS $\mathrm{AP}$ wire. In this study we only consider the dependence of $J_{e}$ on the magnitude $B$ of the critical current density: the dependence on the direction of the field is not considered. The different lines correspond to different temperatures $T$. The data shown here correspond to the engineering current density. 2b Vacodym [29] NdFeB permanent magnet $M_{\|}-H_{\|}$hysteresis loop (blue curve), and $B_{\|^{-}}$ $H_{\|}$loop (red curve). The remanent flux density $B_{r}$, the intrinsic coercivity $H_{c i}$ and the normal coercivity $H_{c b}$ are indicated.

It is worth mentioning that the simulations for the Helmholtz coil have been performed using a 2D axisymmetric model, while the simulations for the finite-length Halbach cylinder can only be performed using a full 3D model.

\subsection{Field-intensity and field-precision estimation}

After performing the simulations, we need to extract information about the strength and precision of the field distribution inside the ROI. For the field-strength, we consider the average 
ROI flux density:

$$
\langle B\rangle_{\mathcal{R}_{\mathrm{ROI}}}=\frac{\int_{\mathcal{R}_{\mathrm{ROI}}} \mathrm{d} V\|\boldsymbol{B}(\boldsymbol{x})\|}{\int_{\mathcal{R}_{\mathrm{ROI}}} \mathrm{d} V}
$$

where $\mathcal{R}_{\text {ROI }}$ denotes the set of points in the ROI. The average flux density $\langle B\rangle_{\mathcal{R}_{\text {ROI }}}$ will simply be denoted by $\langle B\rangle$. Similarly we introduce $\langle\boldsymbol{B}\rangle_{\mathcal{R}_{\mathrm{ROI}}}$, also denoted as $\langle\boldsymbol{B}\rangle$ :

$$
\langle\boldsymbol{B}\rangle_{\mathcal{R}_{\mathrm{ROI}}}=\frac{\int_{\mathcal{R}_{\mathrm{ROI}}} \mathrm{d} V \boldsymbol{B}(\boldsymbol{x})}{\int_{\mathcal{R}_{\mathrm{ROI}}} \mathrm{d} V}
$$

The magnetic field precision will be evaluated by the figure of merit $\Delta \in[0,1]$, defined by:

$$
\Delta=\frac{\int_{\mathcal{R}_{\mathrm{ROI}}} \mathrm{d} V\|\boldsymbol{B}(\boldsymbol{x})-\langle\boldsymbol{B}\rangle\|^{2}}{\int_{\mathcal{R}_{\mathrm{ROI}}} \mathrm{d} V\|\boldsymbol{B}(\boldsymbol{x})\|^{2}}
$$

As the field precision increases, $\Delta \rightarrow 0^{+}$. Because of symmetry, for the Helmholtz case $\langle\boldsymbol{B}\rangle$ is oriented along the $z$ direction, and for the Halbach case along the $x$ direction. Clearly $\boldsymbol{B}(\boldsymbol{x})-\langle\boldsymbol{B}\rangle$ is the field distortion. Therefore $\Delta$ can be interpreted as the fraction of the ROI magnetic energy stored into the field distortion. In other words $\Delta$ is a relative measure of the field-distortion; for example $\Delta=10^{-6}$ means that $1 \mathrm{ppm}$, (one part per million) of the total magnetic energy is associated with the field distortion; in this case we will use a logarithmic notation and write: $\log _{10}(\Delta)=-6$.

The figure of merit $\Delta$ can also be calculated from the coefficients $c_{k}$ of expansion of $\boldsymbol{B}(\boldsymbol{x})$ into spherical or cylindrical harmonics $27, \boldsymbol{B}_{k}(\boldsymbol{x})$ :

$$
\boldsymbol{B}(\boldsymbol{x})=\sum_{k} c_{k} \boldsymbol{B}_{k}(\boldsymbol{x})
$$

By assuming that the harmonic functions are normalized with respect to the region $\mathcal{R}_{\mathrm{ROI}}$, and that $k=0$ corresponds to the desired field component, the quantity $\Delta$ also corresponds to the normalized squared sum of the undesired coefficients:

$$
\Delta=\frac{\sum_{k=1, \ldots, \infty}\left|c_{k}\right|^{2}}{\sum_{k=0, \ldots, \infty}\left|c_{k}\right|^{2}}
$$

In Sec. 3 we will consider $\langle B\rangle$ and $\Delta$ to evaluate field strength and field precision, respectively. It should however be stressed that the real value of $\Delta$ (in particular) is affected by many details which are not considered here. For the superconducting case they include: the real geometry of the coils, subdivision into filaments, screening currents, active and passive shimming, and others. For the permanent magnet case one must consider the segmentation, shimming, remanence variation between various blocks, and others. All these details cannot be known during the exploratory stage which is the scope of this paper. For the purpose of a feasibility study it is sufficient to estimate the order magnitude of field precision and compare the different alternatives.

\subsection{Cost estimation}

For the cost estimation we follow the procedure outlined in Ref. 9]. All the cost quantities will be expressed in euros. It should be stressed, that the procedure outlined here provides a rather rough estimate of the total cost of the main magnetic field source of the device. Clearly, the precise cost cannot be estimated without specifying in much more details the design of the 
device under study. Nevertheless, it is a reasonable estimate of the cost range, and can thus be used as a guideline. The present work is to be intended as a feasibility study comparing two radically different alternatives over a wide range of requirements.

Superconductor - For the superconducting machine, the total cost $C$ is the sum of three terms:

$$
C=C_{\text {Mat. }}^{(\text {Base })}+C_{\text {Cryo }}^{(\text {Base }}+C_{\text {Cryo }}^{(\text {Run })}
$$

Here $C_{\text {Mat. }}^{\text {(Base) }}$ is the cost of the superconducting material, $C_{\text {Cryo }}^{(\text {Base }}$ is the base cost of the cryocooler, and $C_{\text {Cryo }}^{(\text {Run })}$ is the operating cost of the cryocooler. The base material cost is simply given by the coil volume $V_{S C}$ multiplied by the cost volume density $c_{S C}$ of the superconducting material:

$$
C_{\text {Mat. }}^{(\text {Base }}=V_{S C} c_{S C}
$$

Following Ref. 9] from 2016, one meter of $4 \mathrm{~mm}$-wide tape costs $C_{S C}^{(0)}=20.28$ euros, and has a thickness of $0.080 \mathrm{~mm}$. The cost per volume $c_{S C}$ is thus $63.375 \times 10^{6}$ euros $/ \mathrm{m}^{3}$. The cryocooler base and operating costs depend on the required cooling power $\dot{\mathcal{Q}}_{C}$ and the coldside temperature $T$. We thus need to estimate the main sources of heat that the cryocooler needs to compensate for. We assume that the low-temperature region $\mathcal{R}_{\text {Cool }}$ is the hollow cylinder having internal radius $R-W / 2$, external radius $R+W / 2$ and axial length $R+W$. This cylinder incorporates both the coils. In this study we decompose the total cooling power $\dot{\mathcal{Q}}_{C}$ in three terms: the conduction heat flowing from the current leads to the superconducting material, denoted by $\dot{\mathcal{Q}}_{\text {Leads }}$, the thermal radiation incoming from the external environment into the low-temperature region, denoted by $\dot{\mathcal{Q}}_{\text {Rad. }}$, and the small heat generated inside the superconductor by Joule effect, denoted by $\dot{\mathcal{Q}}_{\text {Joule }}$. We thus have:

$$
\dot{\mathcal{Q}}_{C}=\dot{\mathcal{Q}}_{\text {Leads }}+\dot{\mathcal{Q}}_{\text {Rad. }}+\dot{\mathcal{Q}}_{\text {Joule }}
$$

Following Ref. 9] we estimate $\dot{\mathcal{Q}}_{\text {Leads }}$ by assuming a heat absorption rate of $\dot{q}=90 \mathrm{~W} / \mathrm{kA}$. The value of $\dot{\mathcal{Q}}_{\text {Leads }}$ is the product of $\dot{q}$ and the total current $I_{\text {Tot. }}$. The current is obtained from the FEM simulations from the number of windings of $4 \mathrm{~mm}$-wide and $80 \mu \mathrm{m}$-thick tape required to entirely fill up the space of each coil. Only a small fraction of $\dot{\mathcal{Q}}_{C}$ is due to $\dot{\mathcal{Q}}_{\text {Leads }}$ or $\dot{\mathcal{Q}}_{\text {Joule }}$; the only significant term is $\dot{\mathcal{Q}}_{\mathrm{Rad}}$. The net radiation heat intake is calculated from Stefan-Boltzmann law:

$$
\dot{\mathcal{Q}}_{\mathrm{Rad} .}=\varepsilon \sigma_{S B}\left(T_{\mathrm{Env} .}^{4}-T^{4}\right) S_{\mathrm{Cool}}
$$

Here $\sigma_{S B}$ is the Stefan-Boltzmann constant, $\varepsilon$ the emissivity, assumed to be $\varepsilon=0.1, T_{\mathrm{Env}}$. $300 \mathrm{~K}$ is the room temperature, and $S_{\text {Cool }}$ is the external surface area of $\mathcal{R}_{\text {Cool }}$.

The Joule dissipation inside the SC is calculated from the superconductor coil volume $V_{S C}$ multiplied by the power density: $\dot{\mathcal{Q}}_{\text {Joule }}=V_{S C} \boldsymbol{J} \cdot \boldsymbol{E}$. The current density $\boldsymbol{J}$ is obtained from the simulations. The electric field is aligned with $\boldsymbol{J}$ and its magnitude can be calculated from the $J$-E power law. Combing Eq. 10 with Eq. 11, we see that the electric field magnitude $E$ is simply given by: $E=E_{c} \lambda^{n}$. From Ref. [12] the exponent $n$ appearing in the power law is $n=28$. In conclusion we have:

$$
\dot{\mathcal{Q}}_{\text {Joule }}=V_{S C} J E_{c} \lambda^{n}
$$

The value of $\dot{\mathcal{Q}}_{\text {Joule }}$ does not depend on the number of windings, but only the coil volume $V_{S C}$.

As was anticipated, the estimate of $\dot{\mathcal{Q}}_{C}$ is necessary for calculating the base and operating costs of the cryocooler. A higher cooling power requires a more powerful and expensive cryocooler. Following Ref. 9], we assume that the base cost is given by:

$$
C_{\text {Cryo }}^{\text {(Base) }}=\dot{\mathcal{Q}}_{C} \times\left(\frac{176 \text { euro }}{W}\right)
$$


Finally, we can calculate the operating cost as:

$$
C_{\text {Cryo }}^{(\text {Run })}=c_{k W h} \frac{\dot{\mathcal{Q}}_{C}}{\eta} \tau
$$

here $\tau$ is the service life of the MRI apparatus (assumed to be 11.5 years), $c_{k W h}=0.088$ euro $/ \mathrm{kWh}$ is the electrical energy cost [9], and $\eta$ is the total efficiency of the device (assumed

to be $5 \%$ ). Overall, the material cost $C_{\text {Mat. }}^{\text {(Base }}$ has the largest impact on the total cost $C$. It is worth mentioning that cryocoolers also require to be serviced approximately every 10000 hours; however, this aspect has a minor impact on the total cost, and therefore is not considered in this cost analysis.

Permanent magnet - For the case of the permanent magnet we estimate the total cost $C$ directly from the magnet volume $V_{P M}$ :

$$
C=V_{P M} c_{P M}
$$

where $c_{P M}=686.400 \times 10^{3}$ euros $/ \mathrm{m}^{3}$ is the cost volume density of the permanent magnet material[9], and $V_{P M}$ is given by: $V_{P M}=\pi L_{z}\left(R_{O}^{2}-R_{I}^{2}\right)$. The cost volume density $c_{P M}$ corresponds to a cost mass density of $\approx 87.7$ euro $/ \mathrm{kg}$.

\section{Results and discussion}

\subsection{Qualitative dependence on the design parameters}

Before addressing the comparison between the superconducting and permanent magnet based systems, it is insightful to discuss qualitatively the two alternatives individually. Therefore, we will begin by presenting the dependence of the three relevant quantities $\langle B\rangle, \Delta$, and $C$ on the various design parameters.

Superconductor - Fig. 3 shows the results for the REBCO-based Helmholtz coil. The field strength, quantified by $\langle B\rangle$, is shown in Fig. 3a as function of $R$ and $W$. Fig. 3b shows the order of magnitude of non-homogeneity, quantified by $\log _{10}(\Delta)$. Fig. $3 \mathrm{c}$ shows the order of magnitude cost, quantified by $\log _{10}(C)$ with $C$ expressed in euros. The red solid lines are the contour corresponding to $T=77 \mathrm{~K}$, and the blue dashed lines correspond to $T=4.2 \mathrm{~K}$.

As shown in Fig. 3a, when the superconducting wire operates at a lower temperature, it can carry more current and thereby the coils are able to generate a stronger field. In all cases, the field strength increases as the side length $W$ of the coils is increased. To a lesser extent $\langle B\rangle$ also increases as the radius of the coil is reduced, i.e. when the field source is brought closer to the ROI.

As shown in Fig. 3b, the field-precision is almost exclusively affected by the radius $R$ of the coil: when the coils are more distant from the ROI, the field distribution inside the ROI is more homogeneous. The operating temperature $T$ and the coil side length $W$ have a very marginal effect on $\Delta$.

The total cost $C$, shown in Fig. $3 \mathrm{c}$. increases primarily with $W$ and to lesser extent with $R$. Since the total cost is mostly due to the materials rather than the operating costs, the cost $C$ is largely independent of the operating temperature $T$. Only for small values of $W$ and $R$, the cost difference due to the operating temperature $T$ is appreciable, and a lower temperature entails a higher cost. As will be discussed in Sec. 3.2, the optimal operating temperature is affected by the field requirements.

Permanent magnet - Fig. 4 shows the results for the NdFeB-based Halbach cylinder. As for Fig. 3, the three panels correspond to field strength, field precision, and cost, respectively. The results are shown as function of external radius $R_{O}$ and axial length $L_{z}$. The black solid 


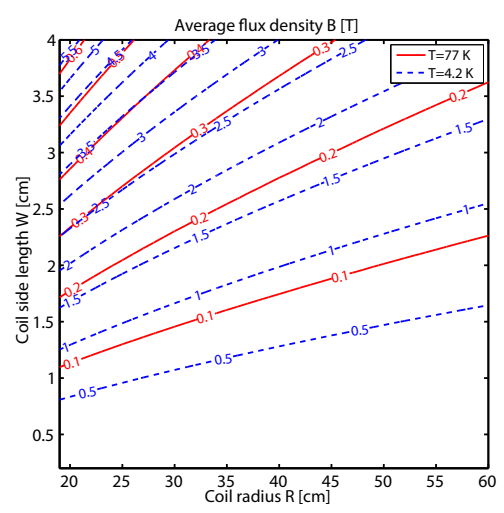

(a) Field strength

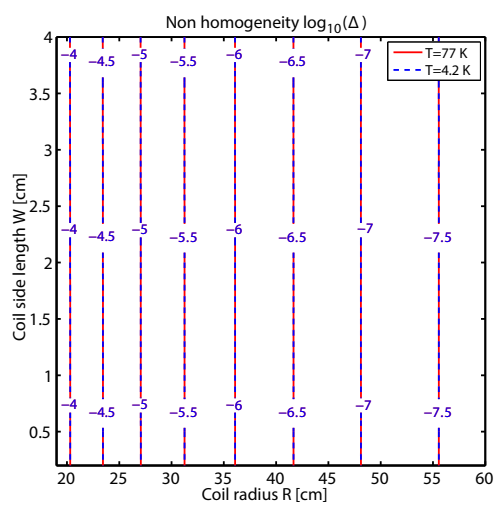

(b) Field precision

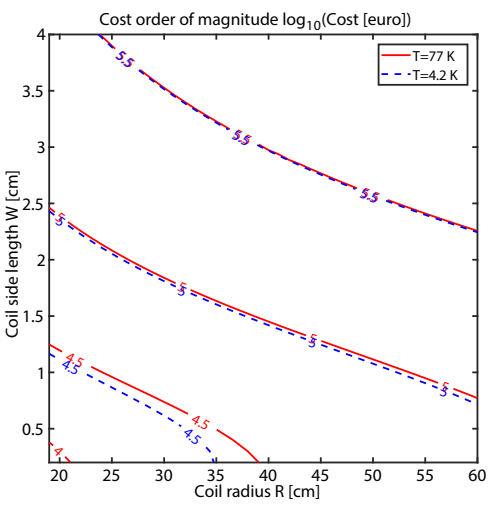

(c) Total cost

Figure 3: Superconducting REBCO-based Helmholtz coil simulation results. The results are plotted as function of radius $R$ and side-length $W$ of the coils. Fig. $3 \mathrm{a}, 3 \mathrm{~b}$, and $3 \mathrm{c}$ show the values of $\langle B\rangle$, $\Delta$, and $C$, respectively. The red solid contour lines correspond to $T=77 \mathrm{~K}$, and the blue dashed contour lines to $T=4.2 \mathrm{~K}$

lines are the contours for the non-linear simulation based on Eq.12 and Fig. 2b For comparison, we also performed a simulation corresponding to the simplified case of a field-independent magnetization defined by $\mu_{0} \boldsymbol{M}=(1.428 \mathrm{~T}) \hat{\boldsymbol{e}}_{\|}$. This value of $\boldsymbol{M}$ corresponds to the remanent magnetization of the non-linear model, i.e. $M_{\|}\left(H_{\|}\right)$for $H_{\|}=0$, (see appendix A.1). The corresponding remanent flux density is indicated in Fig. 2b with the symbol $B_{r}$. The results of this simplified (linear) model are shown in Fig. 4 as red dashed lines. In both cases the magnet scale factor is $\sigma=1$. The blue dotted lines instead correspond to a non-linear simulation for $\sigma=1.25$.

The purpose of the linear simulation is to highlight the effect of finite-coercivity by comparison with the non-linear simulation. This comparison provides an indication of the range of parameters for which a linear simulation would be sufficiently accurate for predicting the behavior of a $\mathrm{PM}$ material with a coercivity of $\approx 1.29 \mathrm{~T}$. Moreover, the linear model gives an estimate of the results that could be expected if a different grade of $\mathrm{NdFeB}$ with much higher coercivity was selected.

As expected from Eq. 2, and confirmed by the results shown in Fig. 4a, the field strength increases when the ratio $R_{O} / R_{I}$ increases. The intensity also increases with $L_{z}$. For relatively short cylinders, i.e. when $L_{z}<R_{O} / 2$, the intensity $\langle B\rangle$ is more significantly affected by $L_{z}$ than it is by $R_{O}$. For parameters giving an average field around $1.5 \mathrm{~T}$ or lower, the linear and non-linear model are consistent. Above this threshold the non-linear model realistically predicts a lower intensity. This decrease is due to the fact that, when the ratio $R_{O} / R_{I}$ of the Halbach cylinder is increased, the inner equatorial regions of the magnet (i.e. $x=0$ and $y \approx R_{I}$ ) experience an increasingly intense demagnetizing field oriented in the opposite direction of their magnetization vector [11. When the magnitude of the demagnetizing field is too large with respect to the coercivity $H_{c}$ of the PM material, non-linear demagnetization occurs since the operating point moves in the steep region of the $M_{\|}\left(H_{\|}\right)$, i.e. after the knee point. This phenomenon cannot be captured by the linear model. As expected, $\langle B\rangle$ is only marginally affected by $\sigma$. In fact, since the governing equations are scale-invariant, increasing $\sigma$ is exactly equivalent to reducing the size of the ROI which is located in a region where the 


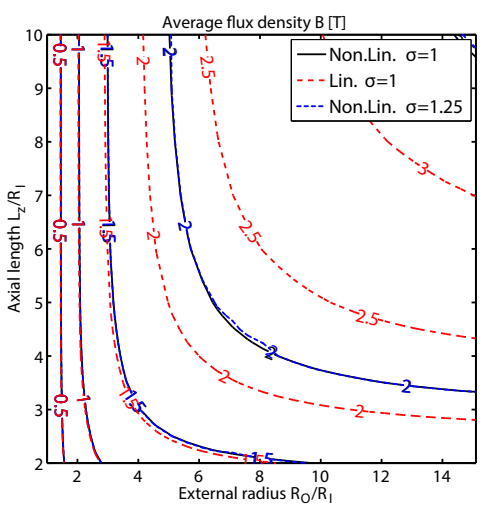

(a) Field strength

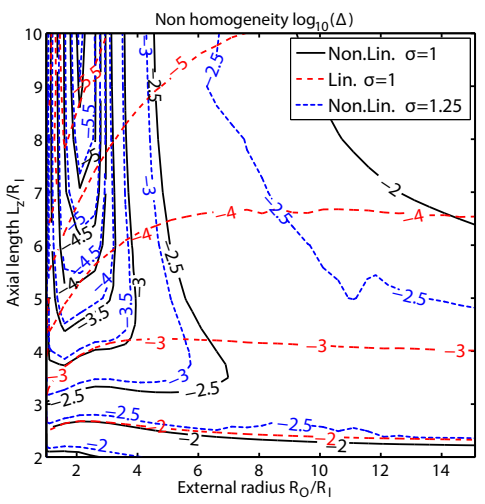

(b) Field precision

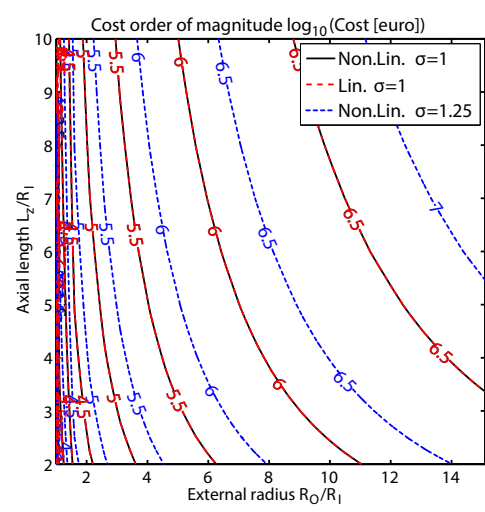

(c) Total cost

Figure 4: Permanent magnet NdFeB-based Halbach cylinder simulation results. The results are plotted as function of outer radius $R_{O}$ and axial length $L_{z}$ of the magnet. Fig. $4 \mathrm{a}$, $4 \mathrm{~b}$, and $4 \mathrm{c}$ show the values of $\langle B\rangle, \Delta$, and $C$, respectively. The red dashed contours correspond to the case of scale factor $\sigma=1$, computed with a linear simulation, the black solid contours to $\sigma=1$ and non-linear simulation, and the blue dotted contours to $\sigma=1.25$ and non-linear simulation.

field magnitude is almost uniform.

The general behaviour of the field precision predicted by the linear model, shown by the red dashed lines in Fig. 4b is that the homogeneity improves as the axial length is increased. This correlation is expected since within the linear model the finite-length is the only source of fielddistortion: an infinitely long cylinder modelled with a linear constitutive relation provides a perfectly uniform field. For long and thin cylinders the value of $R_{O}$ also affects the homogeneity. The results obtained with a non-linear constitutive relation are less trivial: as shown by the black solid contour lines, the two models give the same result for small values of $L_{z}$. However, as $L_{z}$ is increased the non-linear simulation predicts a different behaviour where increasing $R_{O}$ beyond $R_{O} / R_{I} \approx 2$ can decrease the field homogeneity. Such an observation can be ascribed to the detrimental effects caused by the stray field and by non-linear demagnetization of the permanent magnet in certain regions of the assembly. The non-linear simulation corresponding to $\sigma=1.25$, indicated by the blue dotted contour lines, shows a similar trend to the case $\sigma=1$, but generally gives a higher homogeneity. This improvement is again due to the fact that the field source is farther away from the ROI.

As discussed in Sec. 2.5 for the permanent magnet case our cost estimate only depends on the total magnet volume. The value of $C$, shown in Fig. 4c thus depends trivially on the three geometrical parameters $R_{I}, R_{O}$ and $\sigma$. The cost increases linearly with $L_{z}$, quadratically with $R_{O}$, and is proportional to $\sigma^{3}$.

It is worth mentioning that the data is more affected by numerical noise for the $3 \mathrm{D}$ simulations of the Halbach cylinder than it is for the 2D simulations of the Helmholtz coil. This is particularly noticeable for the estimation of $\Delta$, see Fig. 4b. A more precise calculation of $\Delta$ would require a finer mesh, thus severely increasing the computational time especially for large value of $\sigma$. 


\subsection{Optimal design parameters and comparison}

As mentioned before, in the case of the superconducting field source we varied three independent parameters, i.e. $R, W$, and $T$, and we computed three figures of merit $\langle B\rangle, \Delta$ and $C$. Applying the same figures of merit for the permanent magnet field source, the three design parameters $R_{O} / R_{I}, L_{z} / R_{I}$ and $\sigma \propto R_{I}$ were considered. The dimensionality of the parameters space can be reduced by one., i.e from $3 \mathrm{D}$ to $2 \mathrm{D}$, by optimizing the cost for a fixed combination of field constraints with respect to the combination of design parameters.

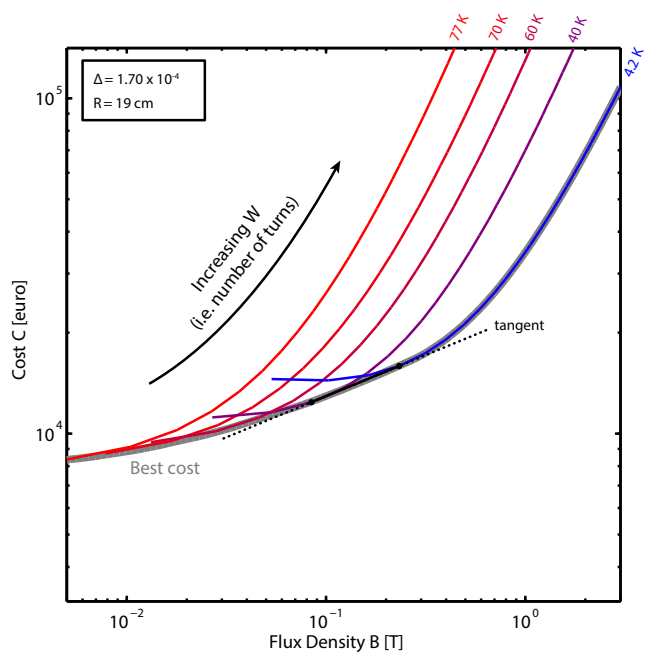

(a) Helmholtz coil - cost optimization

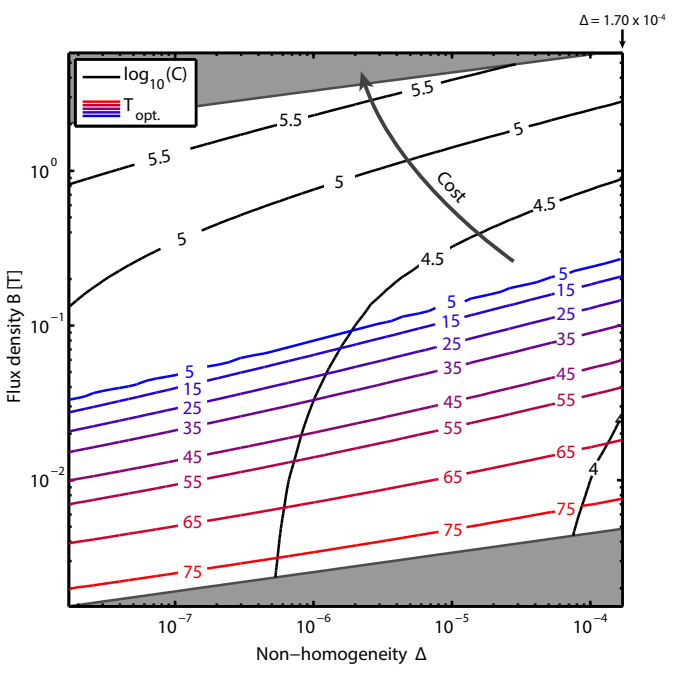

(b) Helmholtz coil - Opt. cost landscape

Figure 5: Superconducting Helmholtz coil cost optimization results. 5a constant radius $R$ curves corresponding to different operating temperatures. All the curves give $\Delta=1.7 \times 10^{-4}$. The optimal cost $C_{\text {opt }}$ can be obtained as the envelope of all the curves, and is indicated by the grey thick line. Numerically, this is obtained by extracting the common tangent between consecutive curves. $5 \mathrm{~b}$ the black contour lines show $\log _{10}\left(C_{\text {opt }}\right)$ as function of $\langle B\rangle$ and $\Delta$. The optimal $T$ is indicated by the contours with colours ranging from blue to red.

Superconductor - Let us first consider the case of the superconducting coil. As mentioned in Sec. 3.1 the field precision is primarily determined by the coil radius $R$. The results presented in Fig. 5a correspond to fixed radius $R=19 \mathrm{~cm}$, leading to non-homogeneity $\Delta=1.7 \times 10^{-4}$. As shown in Tab. 1, $19 \mathrm{~cm}$ is the smallest value of radius that has been simulated. The different lines show the total cost $C$ as function of $\langle B\rangle$ for different values of $T$, which correspond to the different colours as indicated by the labels. Increasing $W$ increases $\langle B\rangle$, as well as $C$. Since the different curves are monotonically increasing and intersecting, the minimum cost is given by the envelope of the family of curves corresponding to the various $T$. As indicated by the black dotted line in Fig. 5a, the envelope can be calculated numerically by considering the mutual tangent between two consecutive curves. The optimal cost $C_{\text {opt }}$ as function of $\langle B\rangle$ is plotted as a thick grey line. The slope of the $C_{\text {opt }}(\langle B\rangle)$ curve is higher in the region where the optimal operating temperature is $4.2 \mathrm{~K}$, i.e. the lowest considered temperature. This behaviour is due to the fact that, when is not possible to further reduce the operating temperature, the only 
way to improve the field is to increase the coil size $W$, which leads to a steeper cost function.

Repeating the same procedure for all values of $R$ shown in Tab. 1 we can calculate the optimal cost as function of $\langle B\rangle$ and $\Delta$. The black contour lines shown in Fig. $5 \mathrm{~b}$ correspond to values of $\log _{10}\left(C_{\mathrm{opt}}(\langle B\rangle, \Delta)\right)$. The contour lines with colours ranging from red to blue indicate the optimal operating temperature. The data shown in Fig. $5 \mathrm{a}$ correspond to the right margin of Fig. $5 \mathrm{~b}$ below $\langle B\rangle=0.3 \mathrm{~T}\left(C=1.7 \times 10^{4}\right.$ euros $)$ the cost increases moderately as the optimal operating temperature decreases, past $0.3 \mathrm{~T}$, the optimal temperature remains $4.2 \mathrm{~K}$ and the cost increases steeply up to $>10^{5}$ euros. The grey-shaded triangular regions on the bottom and top of the figure are outside the range of simulated parameters.

From Fig. $5 \mathrm{~b}$ we can notice that when higher field strength or field precision is required, the optimal operating temperature is lower. Only when $\langle B\rangle<10^{-2} \mathrm{~T}$ would be convenient to operate the coil at $77 \mathrm{~K}$. Within the range of simulated parameters we can reach a maximum field of $5.78 \mathrm{~T}$ with $\Delta=1.7 \times 10^{-4}$, and an estimated cost of $2.4 \times 10^{5}$ euros (corresponding to the top-right corner of Fig. $5 \mathrm{~b}$. Alternatively, the best precision of $\Delta=1.7 \times 10^{-8}$ corresponds to $\langle B\rangle=2.03 \mathrm{~T}$, and an estimated cost of $8.39 \times 10^{5}$ euros (top-left corner).

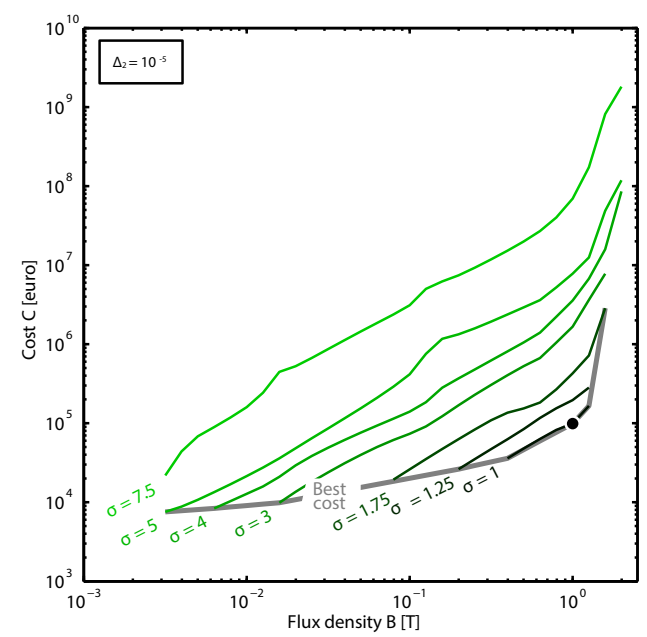

(a) Halbach cylinder - cost optimization

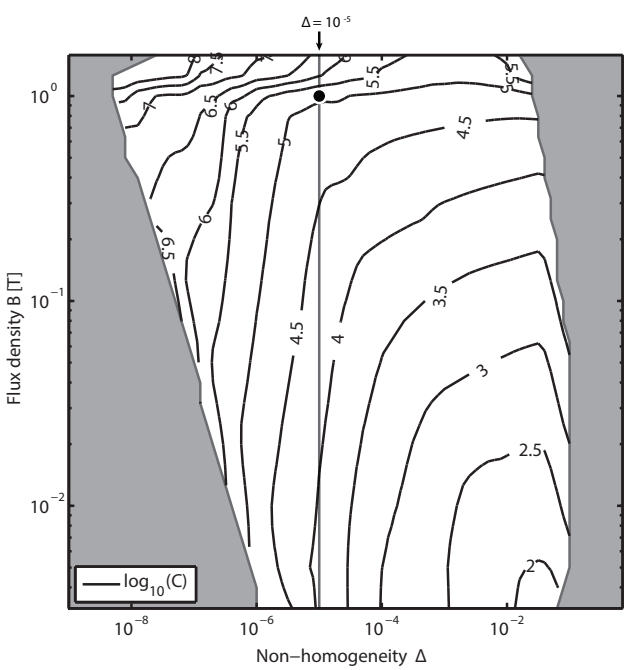

(b) Halbach cylinder - Opt. cost landscape

Figure 6: Permanent magnet NdFeB-based Halbach cylinder cost optimization results. 6a. constant $\Delta$ curves corresponding to different values of $\sigma$ (i.e. different $R_{I}$ ). These curves have been calculated by interpolating the original data. The optimal cost $C_{\text {opt }}$ can be obtained by minimizing $C$ with respect to $\sigma$ for each value of $\langle B\rangle$, and is indicated by the grey thick line. Numerically, this is estimated from the common convex hull of all the curves. 6b; the black contour lines show $\log _{10}\left(C_{\text {opt }}\right)$ as function of $\langle B\rangle$ and $\Delta$.

Permanent magnet - For the case of the Halbach cylinder we followed a similar procedure, with a few differences. As we can see from Fig. $4 \mathrm{~b}$ for the Halbach case $\Delta$ does not have a simple dependence on just one of the parameters. In order to calculate the optimal cost function $C_{\text {opt }}(\langle B\rangle, \Delta)$ we have to interpolate the simulation result on a common grid of $\langle B\rangle, \Delta$ values. Fig. 6a shows a series of $C_{\text {opt }}(\langle B\rangle)$ slices for different values of $\sigma$, indicated by the labels, and constant $\Delta=10^{-5}$. Another difference with respect to the Helmholtz case is that the various 
curves, corresponding to different values of $\sigma$, do not intersect with each other. To overcome this difficulty we compute the lowest cost point for each value of $\langle B\rangle$ by calculating the convex hull of the set of all data points plotted in Fig. 6a. The lower branch of the convex hull is plotted as a thick grey line. As we can see from the figure, this line is a realistic estimation of the optimal cost function $C_{\text {opt }}(\langle B\rangle)$. This curve is characterized by a low-steepness region for $\langle B\rangle \lesssim 0.4 \mathrm{~T}$ : in this region $\langle B\rangle$ can be improved by reducing $\sigma$, i.e. scaling down the magnet so that its inner border is closer to the ROI. After this threshold the cost increases quite steeply with $\langle B\rangle$.

Again, we can repeat the same procedures for different values of $\Delta$ until we calculate the optimal cost function $C_{\mathrm{opt}}(\langle B\rangle, \Delta)$, which is shown in Fig. 6b. As in Fig. 5b the black contour lines indicate different values of $\log _{10}\left(C_{\mathrm{opt}}\right)$. The grey shaded regions on the left and right border of the canvas are outside the range of simulated parameters. Notice that when the field requirement are very relaxed, the cost estimation of the permanent magnet machine is very low, e.g. $C<10^{3}$ euros. For these extreme cases the volume $V_{P M}$ of the permanent magnet is very small, and it would not be realistic to estimate the cost by only considering $V_{P M}$. The slice shown in Fig. 6a is indicated in Fig. 6b by the vertical grey line. We can see that the contour lines of Fig. 6b are consistent with the grey curve shown in Fig. 6a the cost increases moderately until $\langle B\rangle$ is below $0.4 \mathrm{~T}$ and then it abruptly increases up to $>10^{6}$ euros. For the Halbach cylinder increasing the average ROI field above $\approx 1 \mathrm{~T}$, or getting a field precision better than $\Delta \approx 10^{-5}$, requires a relatively expensive machine, i.e. $C \gtrsim 10^{5}$ euros. This for example would mean that with $\langle B\rangle=1 \mathrm{~T}, \Delta=10^{-5}$, corresponding to the black dot in Fig. 6a and Fig. 6b, the cylinder would have scale factor $\sigma=1$, inner radius $R_{I}=19 \mathrm{~cm}$, $V_{P M}=0.144 \mathrm{~m}^{3}$, and a cost $C=9.89 \times 10^{4}$ euros.

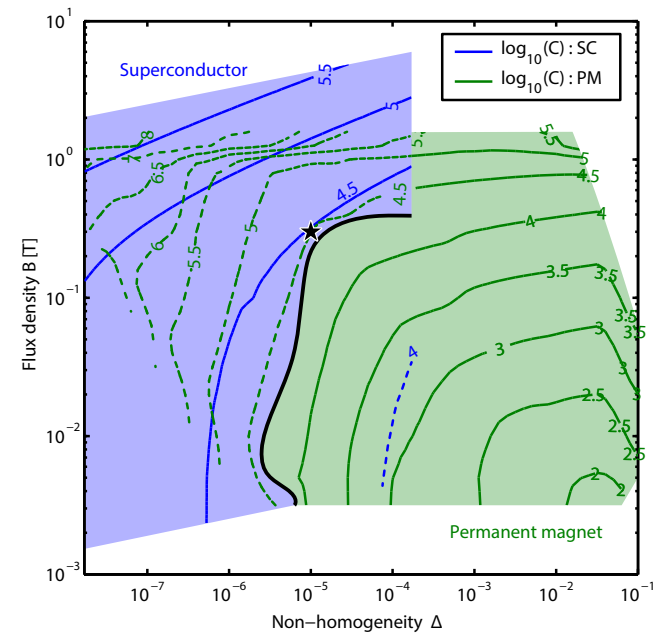

Figure 7: Comparison between the cost of the two systems. The figure combines the data of Fig. $5 \mathrm{~b}$ and Fig. $6 \mathrm{~b}$. The blue and green $\log _{10}\left(C_{\mathrm{opt}}\right)$ contour lines correspond to the SC system and the PM system, respectively. The SC system is the most advantageous choice for combinations of $\langle B\rangle$ and $\Delta$ corresponding to the blue-shaded region, and the PM is more convenient over the green-shaded region. The thick black line shows the boundary between the two regions.

Comparison - We can now compare the two alternative magnetic systems by combining 
the data shown in figures $5 \mathrm{~b}$ and $6 \mathrm{~b}$ The combined results are shown in Fig. 7. The superconducting cost contours are plotted as blue lines and the permanent magnet contours by green lines. The shade indicates in which region one option is more convenient than the other one. The thick black line separates the two regions. The permanent magnet is the most convenient choice for low field strength and relaxed field-precision requirements.

In general, the results shown in Fig. 7 span the range of field requirements necessary for MRI systems: $\langle B\rangle \approx 0.1-3 \mathrm{~T}$, and $\Delta \approx 10^{-6}-10^{-5}$, i.e. a few ppm of field distortion [30. However, it is important to keep in mind that the field-homogeneity is affected by several factors not considered in our comparative analysis, such as the specific design of the magnetic systems, active and passive shimming, PM segmentation, etc.

It is insightful to consider in detail a specific example. With the field requirements $\langle B\rangle=$ $0.3 \mathrm{~T}$, and $\Delta=10^{-5}$, the cost of the PM and SC systems are nearly equal: $\log _{10}(C[$ euro $]) \approx 4.5$, i.e. $C=3.2 \times 10^{4}$ euros. These conditions correspond to the black star shown in Fig. 7 . The SC system is approximately characterized by the following set of parameters: $W=0.75 \mathrm{~cm}$, $R=27 \mathrm{~cm}, T=4.2 \mathrm{~K}$. The PM system satisfying the same field requirements is approximately characterized by the following set of parameters: $\sigma=1.25, R_{I}=\sigma \times 10 \mathrm{~cm}=12.5 \mathrm{~cm}$, $R_{O}=1.5 R_{I}, L_{z}=8 R_{I}$.

HTS tape technology is currently employed in ultra high field magnets, for both MRI and NMR. For these applications, though the tape geometry does pose several challenges with respect to field homogeneity and stability due to screening current decay and complex persistent mode operation. The former can be mitigated by subdividing the HTS tape into a number of fine filaments 1, 2, 4, 3. Persistent mode operation requires manufacturing of superconducting joints between multiple HTS tapes (REBCO-based) and it has been demonstrated [5, 6] that such technology is greatly advancing. HTS tape technology is therefore now considered technically feasible for the before mentioned applications.

\section{Conclusion}

We conducted a feasibility investigation comparing a REBCO superconducting coil with a NdFeB permanent magnet assembly as field source for the main magnet of a small-scale MRI scanner. As prototypical geometries we choose the Helmholtz coil arrangement for the superconductor and the Halbach cylinder for the permanent magnet. For each case, we focus on three fundamental design parameters: coil radius, coil thickness and operating temperature for the SC system and inner radius, outer radius and axial length for the PM system. FEM analysis has been employed to calculate the magnetic field distribution generated by the systems for a wide range of parameters' values. Field strength $\langle B\rangle$, field precision $\Delta$ and total cost $C$ have been computed for each case, as these quantities have been identified as the three most important indicators of whether a certain combination of parameters is or is not feasible. Thus we reduced the dimensionality of the parameter's space by optimizing the cost for a fixed combination of $\langle B\rangle$ and $\Delta$. For the case of the superconducting system we can thus obtain the optimal operating temperature for each configuration, while for the permanent magnet case we determine the optimal value of inner radius. Finally, the SC and PM alternatives are compared to each other.

Overall, the superconducting Helmholtz coil is the more convenient choice when the requirements on field-precision are high. The permanent magnet Halbach cylinder can be very convenient when both the field requirements are not too demanding. In both cases there is a trade-off between field strength and field-precision for a given total cost. Considering the representative target field magnitude of $0.3 \mathrm{~T}$, the permanent magnet would be optimal only until $\Delta \approx 10^{-5}$, when higher precision is necessary it would be more convenient to employ the superconductor field-source. 
The systematic procedure outlined in the present study could be expanded or applied to more specific designs. The optimization of $\langle B\rangle$ and $\Delta$ with respect to the operating temperature could be performed for more advanced coil arrangements, such as the magnetic systems discussed in Ref. [28]. Similarly, it would be interesting to extend the present analysis to segmented or iron-yoked permanent magnet systems.

\section{Acknowledgments}

The authors would like to thank Professor Michael Sumption, Department of Materials Science and Engineering, The Ohio State University, for the constructive comments regarding the fieldprecision analysis.

The work of A. Insinga and A. C. Wulff was supported by the ATOMIS DFF-Research Project which is funded by the Independent Research Fund Denmark (project ID DFF-611100252).

\section{A Appendix}

\section{A.1 Constitutive relations model functions}

Superconductor - The data sets for the superconductor $J_{c}(B, T)$ relation (Fig. 2a) and the permanent magnet $M-H$ relation (Fig. 2b] have been extracted from Ref. [12] and Ref. 29], respectively.

We decided to fit the $J_{c}(B, T)$ data with the following model function:

$$
J_{c}(B, T)=J_{c 0} \times f_{B}(B) \times f_{T}(T)
$$

Where the non-dimensional functions $f_{B}(B)$ and $f_{T}(T)$ are defined as:

$$
\begin{gathered}
f_{B}(B)=\frac{1}{1+\left(B / B_{0}\right)^{\alpha}} \stackrel{B \rightarrow 0}{\longrightarrow} 1 \\
f_{T}(T)=\frac{\varepsilon\left(1+(T / \gamma)^{2}\right)-1}{(\varepsilon-1)\left(1+(T / \gamma)^{2}\right)} \stackrel{T \rightarrow 0}{\longrightarrow} 1
\end{gathered}
$$

The function $f_{B}$ is a rational function of the flux density norm $B$. As long as $B_{0}>0$, the limit of $f_{B}$ for $B \rightarrow 1$ is 1 . The exponent $\alpha$ and the parameter $B_{0}$ determine how quickly $f_{B}$ decreases as $B$ increases.

The function $f_{T}$ is a Lorentz distribution with centre in $T=0$, full width at half-maximum $2 \gamma$, vertically translated by $\epsilon$ and then re-normalized so that $f_{T}(T) \rightarrow 1$ as $T \rightarrow 0$.

We assume that the parameters $B_{0}$ and $\alpha$ appearing in the expression of $f_{B}$ are also temperature-dependent. The temperature dependence can be modelled by a third degree polynomial:

$$
\begin{aligned}
& B_{0}(T)=\sum_{k=0}^{3} \beta_{k} T^{k} \\
& \alpha(T)=\sum_{k=0}^{3} \alpha_{k} T^{k}
\end{aligned}
$$


The parameters' values giving the best match with the data-points of Ref. [12] are:

$$
\begin{array}{ccc}
J_{c 0}=2.6931 \mathrm{MA} / \mathrm{cm}^{2} ; & \beta_{0}=0.2049 \mathrm{~T} ; & \alpha_{0}=0.6924 \\
\gamma=15.1217 \mathrm{~K} ; & \beta_{1}=-2.483310^{-2} \mathrm{~T} / \mathrm{K} ; & \alpha_{1}=-2.676110^{-3} \mathrm{~K}^{-1} \\
\varepsilon=0.0251 ; & \beta_{2}=2.629410^{-3} \mathrm{~T} / \mathrm{K}^{2} ; & \alpha_{2}=5.693210^{-4} \mathrm{~K}^{-2} \\
& \beta_{3}=-2.805810^{-5} \mathrm{~T} / \mathrm{K}^{3} ; & \alpha_{3}=-4.699410^{-6} \mathrm{~K}^{-3}
\end{array}
$$

It should be stressed that Eq. 26 represents the engineering current density (assuming wire thickness $80 \mu \mathrm{m}$ ), while Ref. [12] gives the total current $I_{c}$ carried by a $4 \mathrm{~mm}$-wide tape. The proportionality factor can be easily calculated.

Permanent magnet - For the permanent magnet we fitted the experimental data with the following model function:

$$
M_{\|}\left(H_{\|}\right)=\left(\frac{M_{0}}{\pi / 2}\right) \operatorname{atan}\left(\frac{H_{\|}-H_{c i}}{\Delta_{H}}\right)
$$

Here $M_{0}$ is the saturation magnetization, (i.e. the maximum value of magnetization corresponding to the limit $\left.H_{\|} \rightarrow+\infty\right), H_{c i}$ represents the intrinsic coercivity (i.e. the intersection of the $M_{\|}\left(H_{\|}\right)$curve with the horizontal axis $M=0$ ), and $\Delta_{H}$ can be interpreted as a horizontal scale factor. The values giving the best match with the data from Ref. [29] are:

$$
\begin{gathered}
\mu_{0} M_{0}=1.4407 \mathrm{~T} \\
\mu_{0} H_{c i}=-1.2957 \mathrm{~T} \\
\mu_{0} \Delta_{H}=0.0181 \mathrm{~T}
\end{gathered}
$$

This set of parameters corresponds to the demagnetization branch of the $M_{\|}\left(H_{\|}\right)$hysteresis loop shown in Fig. 2b.

\section{References}

[1] A. C. Wulff, M. Solovyov, F. Gömöry, A. B. Abrahamsen, O. V. Mishin, A. Usoskin, A. Rutt, J. H. Lundeman, K. Thydén, J. B. Hansen and J.-C. Grivel, Two level undercutprofile substrate for filamentary YBa2Cu3O7 coated conductors, Supercond. Sci. Technol., vol. 28, May 2015, Art. ID. 072001.

[2] A. C. Wulff, J. H. Lundeman, J. B. Hansen, O. V. Mishin, Y. Zhao, R. Mohajeri and J.-C. Grivel, A two level undercut-profile substrate for chemical-solution-based filamentary coated conductors, IEEE Trans. on Appl. Supercond., vol. 26, no. 3, Apr. 2016, Art. ID. 6601604 $1-4$.

[3] A. R. Insinga, M. Solovyov, A. Usoskin, A. Rutt, U. Betz, J. H. Lundeman, A. B. Abrahamsen , J.-C. Grivel , F. Gömöry, and A. C. Wulff, Lift-Factor Analysis of Multifilamentary Coated Conductor Produced Using Two Level Undercut-Profile Substrates, IEEE Trans. on Appl. Supercond., vol. 29, no. 5, Aug. 2019, Art. ID. 8200704 1-4.

[4] A. R. Insinga , A. Sundaram, D. W. Hazelton, V, M. Rodriguez Zermeno, A. B. Abrahamsen, Y. A. Opata, J.-C. Grivel, J. H. Lundeman, A. E. Ryming, F. Schweer-Gori, and A. C. Wulff, Two Level Undercut-Profile Substrate-Based Filamentary Coated Conductors Produced Using Metal Organic Chemical Vapor Deposition, IEEE Trans. on Appl. Supercond., vol. 28, no. 4, Jun. 2018, Art. ID. 6601705 1-5. 
[5] A. V. Naumov, D. N. Diev, A. N. Dinisilov, O. P. Anashkin, V. E. Keilin, I. A. Kovalev, V. V. Lobyntsev, V. I. Scherbakov, D. I. Shutova, and V. E. Sytnykov, Current leads and joints for HTS DC cable of $2.5 \mathrm{kA} 20 \mathrm{kV}$, AIP Conference Proceedings, vol. 1573, no. 1, 2014, 1694-1699.

[6] J. S. Murtomäki, G. Kirby, J. van Nugteren, P. Contat, O. Sacristan-de-Frutos, J. Fleiter, F. Pincot, G. de Rijk, L. Rossi, J. Ruuskanen, A. Stenvall, and F. J. Wolf, $10 \mathrm{kA}$ Joints for HTS Roebel Cables, IEEE Trans. on Appl. Supercond., vol. 28, no. 3, Apr. 2018, Art. ID. 4801406 1-6.

[7] V. M. R. Zermeno, A. B. Abrahamsen, N. Mijatovic, B. B. Jensen and M. P. Sørensen, Calculation of alternating current losses in stacks and coils made of second generation high temperature superconducting tapes for large scale application, J. Appl. Phys., vol. 114, no. 17, Nov. 2013, Art. ID. 173901 1-8.

[8] E. Pardo, J. Šouc and L. Frolek, Electromagnetic modelling of superconductors with a smooth current-voltage relation: variational principle and coils from a few turns to large magnets, Supercond. Sci. Technol., vol. 28, no. 4, Feb. 2015, Art. ID. 044003.

[9] R. Bjørk, K. K. Nielsen, C. R. H. Bahl, A. Smith, and A. C. Wulff, Comparing superconducting and permanent magnets for magnetic refrigeration, AIP Advances, vol. 6, no. 5, 2013, Art. ID. 056205 1-7.

[10] J. V.M. McGinley, M. Ristic, and I. R. Young, A permanent MRI magnet for magic angle imaging having its field parallel to the poles, J. Magn. Reson, vol. 271, 2016, 60 - 67.

[11] A.R. Insinga, C.R.H. Bahl, R. Bjørk, and A. Smith, Performance of Halbach magnet arrays with finite coercivity, J. Magn. Magn. Mater., vol. 407, Feb. 2016, 369-376.

[12] R. Nakasaki, P. Brownsey, A. Sundaram, Y. Zhang, D. Hazelton, H. Sakamoto, and T. Fukushima, Progress of $2 G$ HTS Wire Development at SuperPower, ASC 2016 Denver, USA, Sept. 2016.

[13] M. Parizh, Y. Lvovsky, and M. Sumption, Conductors for commercial MRI magnets beyond NbTi: requirements and challenges, Supercond. Sci. Technol., vol. 30, no. 1, 2017, Art. ID. 014007.

[14] G. Giovannetti, F. Frijia, A. Flori, and D. Montanaro, Design and Simulation of a Helmholtz Coil for Magnetic Resonance Imaging and Spectroscopy Experiments with a $3 T$ MR Clinical Scanner, Appl. Magn. Reson., vol. 50, no. 9, 2019, 1083-1097.

[15] X. He, R. Yuan, B. Keong Li, and Y. Hou, The Design of an Open MRI 4-Channel Receive-Only Phased Array Knee Coil, Appl. Magn. Reson., vol. 47, no. 5, 2016, 499-510.

[16] Z. Cai, R. H. Clarke, B. A. Glowacki, W. J. Nuttall, and N. Ward, Ongoing ascent to the helium production plateau -Insights from system dynamics, Resources Policy, vol. 35, no. 2, 2010, Art. ID. 014007 77-89.

[17] R. A. Slade, B. J. Parkinson, and R. M. Walsh, Test Results for a 1.5 T MRI System Utilizing a Cryogen-free YBCO Magnet, IEEE Trans. on Appl. Supercond., vol. 24, no. 3, Jun. 2014, 1-5.

[18] R. A. Slade, B. J. Parkinson, and R. M. Walsh, Research and Development of the High Stable Magnetic Field ReBCO Coil System Fundamental Technology for MRI, IEEE Trans. on Appl. Supercond., vol. 24, no. 4, Jun. 2017, Art. ID. 4400604 1-4.

[19] Z. Charifoulline, Residual Resistivity Ratio (RRR) Measurements of LHC Superconducting NbTi Cable Strands IEEE Trans. on Appl. Supercond., vol. 16, no. 2, Jun. 2006, 1188-1191.

[20] M.N. Wilson. 100 Years of Superconductivity and 50 Years of Superconducting Magnets, IEEE Trans. on Appl. Supercond., vol. 22, no. 3, Jun. 2012, Art. ID. 3800212 1-12. 
[21] Y. Lvovsky, E. Wolfgang Stautner, and T. Zhang, Novel technologies and configurations of superconducting magnets for MRI, Supercond. Sci. Technol., vol. 26, no. 9, 2013, Art. ID. 093001 .

[22] D. M. Petković, M D. Radić Generalization of Helmholtz Coil Problem, Serb. J. Electr. Eng, vol. 12, no. 3, 2015, 375-384.

[23] G. Moresi, and R. Magin, Miniature permanent magnet for table-top NMR, Concepts in Magnetic Resonance Part B-magnetic Resonance Engineering, vol. 19B, no. 1, 2003, 35-43.

[24] C. Qiaoyan, Z., Guangcai, X. Yajie, and Y. Xiaodong, Design and simulation of a multilayer Halbach magnet for NMR, Concepts in Magnetic Resonance Part B-magnetic Resonance Engineering, vol. 45, no. 3, 2015, 134-141.

[25] K.Halbach, Design of permanent multipole magnets with oriented rare earth cobalt material, Nucl. Instr. Meth. Phys. Res. B, vol. 169, no. 1, 1980, 1-10 .

[26] M. Katter, Angular dependence of the demagnetization stability of sintered $\mathrm{Nd}-\mathrm{Fe}-\mathrm{B}$ magnets, vol. 41, no. 10, 2005, 3853-3855.

[27] J. D. Jackson, Classical Electrodynamics, 3rd Edition, John Wiley and Sons, 1998.

[28] C. Coillot, E. Nativel, M. Zanca, and C. Goze-Bac, The magnetic field homogeneity of coils by means of the space harmonics suppression of the current density distribution, J. Sens. Sens. Syst., vol. 5, no. 5, 2016, 401-408.

[29] Vacuumschmelze GmbH \& Co, KG.Pd002, Rare-Earth Permanent Magnets Vacodym/Vacomax, 2014.

[30] R.G. Sharma, Superconductivity, Basics and Applications to Magnets, Springer Series in Materials Science, 2015. 